\title{
Cooperative Governance: What Impact on the Marketing of Saffron? Case of Saffron's Cooperatives in the Area of Taliouine in Morocco
}

\author{
Zineb Merouah \\ Abdelaziz Bendou \\ Allal Achaba \\ The National School of Commerce and Management of Agadir. \\ Ibn Zohr University, Morocco. \\ E-mail: zineb.merouah@edu.uiz.ac.ma, zineb.merouah@gmail.com
}

\author{
Doi:10.5901/mjss.2013.v4n10p681
}

\begin{abstract}
Cooperatives are major players in the rural areas as they generate income for members and contribute to sustainable socioeconomic development of their territory. Good governance is now recognized as a major factor in organizational effectiveness. The peculiarities of cooperative organizations induce a specific mode of governance to reconcile cooperative principles and the economic logic of the market especially in the international context. This paper aims to present the results of a research whose objective is to show the impact of the governance structure of cooperatives on the marketing of the saffron product of Taliouine region (also known as the country of saffron: a local product with high added value) in Morocco. Our research focuses on the analysis of the nature and components of the structure of governance in saffron cooperatives and the evaluation of their implications on their marketing practices of saffron. This research is conducted by means of semi-structured interviews with presidents and members of the cooperatives of this area.
\end{abstract}

Keywords: Cooperative Governance, Saffron, Marketing.

\section{Introduction}

Dans le cadre du développement durable des régions cloisonnées, les coopératives se présentent comme un instrument efficace pour la promotion de la croissance économique au niveau de ces régions. En effet, les coopératives participent à l'amélioration de la situation socio-économique de ses adhérents. Toutefois, elles sont confrontées à une multitude de problèmes, tels que la mauvaise gestion ou les déficits financiers. A cet effet, ces entités solidaires ont compris que le développement de leur productivité, dépend essentiellement de la mise en place d'un système de gouvernance efficace. Ce régime permet, ainsi, de revoir en permanence le système de gestion des coopératives, à travers une surveillance continue des résultats obtenus.

Notre article s'intéresse à la gouvernance des coopératives safranières de Taliouine, et plus particulièrement à la contribution de cette composante dans la valorisation de la commercialisation du Safran. L'objectif de notre texte est d'étudier l'efficacité du système de gouvernance au sein des coopératives. On s'attachera plus particulièrement à étudier comment la mise en place d'une gouvernance efficace contribue à l'augmentation du chiffre d'affaires de ces entités solidaires.

Premièrement, nous passons en revue les théories organisationnelles à l'origine de l'émergence de la gouvernance dans la littérature des firmes. Par la suite, nous présentons les principes assurant une gouvernance efficace au sein des organisations. En complément à notre étude sur la gouvernance, nous mettons l'accent sur les coopératives au Maroc à travers une lecture détaillée de la loi $n^{\circ}$ 112-12 fixant le statut général des coopératives. La deuxième partie de notre texte, est consacrée à la présentation des résultats de notre étude de l'efficacité du système de gouvernance au sein des coopératives du safran de Taliouine ainsi que de les réponses obtenues à notre objectif de recherche qui est le rôle de la bonne gouvernance dans la promotion de la commercialisation du safran. 


\section{Cadre Conceptuel}

\subsection{L'émergence de la gouvernance dans les théories organisationnelles}

Dans le cadre d'une sphère micro-économique en pleine mutation, le tissu économique est de plus en plus mondialisé. En effet, l'ouverture des capitaux des firmes au marché boursier, a permis de collecter des nouvelles ressources financières par lesquelles l'expansion de ces entités est garantie. Par ailleurs, cette initiative a également créé une multiplicité de propriétaires d'une même firme. A cet effet, il est tout à fait légitime de déléguer à une entité indépendante le droit de manager la firme. Cette nouvelle situation micro-économique, a été le catalyseur de l'émergence de plusieurs théories organisationnelles qui avaient pour objectif de schématiser la relation entre les actionnaires et les managers. Nous allons passer en revue les principales théories qui ont marqué cette relation et qui ont participé à son développement, de telle manière à protéger les droits des propriétaires.

Selon Jensen et Meckling (1976), une relation d'agence est un contrat par lequel une ou plusieurs personnes (le Principal) engage une autre personne (l'Agent) afin d'agir en son nom et qui implique la délégation d'un droit de décision (autorité) à l'Agent. Deux parties sont alors définies, la première est constituée des propriétaires qui sont les bailleurs de fonds, tandis que la seconde est constituée des managers qui ont le rôle de gérer ses fonds, et de prendre les décisions afin de développer cette richesse. En effet, toutes les décisions stratégiques seront prises par cette seconde partie. D'autant plus, que les managers ont un accès à l'information contrairement aux propriétaires qui ne sont au courant que des informations rapportées par les décideurs. Cette situation évoque des interrogations sur l'efficacité de cette relation propriétaires - managers, en prenant en considération un élément crucial à cette réflexion, à savoir les intérêts de chacune des deux parties. II est à noter que les objectifs du principal et de l'agent ne convergent pas nécessairement dans la même direction. En effet, les propriétaires sont plus intéressés par la maximisation du profit, tandis que les managers portent également leur attention à détenir plus de pouvoir décisif. Par conséquent, cette divergence d'intérêts induit à la faiblesse de ce système sociétal. En effet, les décisions prises par les managers pourraient nuire à la société, et impérativement aux droits des propriétaires. Ce résultat nous amène à pousser notre réflexion sur les éléments qui pourront consolider cette relation et éventuellement minimiser ce conflit d'intérêts. II est donc impératif de trouver un terrain d'entente qui fournira une liberté d'agir aux décideurs et au même temps, qui protègera les intérêts des propriétaires. La logique interpelle l'existence, d'une tierce partie, ayant pour rôle de garantir les droits des propriétaires, à savoir un comité de contrôle du travail et des décisions des managers.

D'autres travaux de recherche ont abouti à la même solution citée plutôt, à savoir la création d'un mécanisme de contrôle. Entre autres, les travaux de Alchian et Demsetz (1972), qui ont considéré l'entreprise comme une équipe, dont chacun des membres est conscient que son intérêt dépend de la survie de l'équipe tout entière. De ce fait, la productivité augmente avec le travail en équipe. Par conséquent, cet élément de coopération est garant, selon les mêmes auteurs, de la protection des intérêts des propriétaires. Toutefois, ils présument l'existence, à la fois, d'un système de contrôle et des mesures incitatives (rémunération des membres) pour garantir une meilleure productivité. Dans le même contexte, Jean Jacques Laffont (2000) préconise la théorie des incitations, qui selon lui, « elle fournit une description précise des contraintes imposées par la décentralisation de l'information sur l'allocation des ressources et décrit les propriétés des optima contraints ». II est à noter également les résultats de recherches de Clarke (1971) et de Groves (1973). Ils proposèrent des mécanismes de taxation qui conduisent les agents à révéler leur information en stratégie dominante. Pour cela, il faut donner à l'agent un transfert qui, à une constante près, lui donne la même fonction objectif que le principal, ici le décideur public. L'agent a alors intérêt à annoncer ses vraies préférences puisqu'il a délégué en quelque sorte la décision au principal qui va maximiser la même fonction objectif que lui. Une autre interprétation est qu'on lui fait payer l'externalité positive ou négative qu'il crée sur l'ensemble des autres agents économique (Laffont, 2000).

A l'issue de ces différentes approches, une nouvelle notion a émergée dans le vocabulaire des entreprises, qui est celle du gouvernement d'entreprise. En effet, la gouvernance est apparue comme une solution définitive aux problèmes de conflit d'intérêts entre les actionnaires et les propriétaires. A cet effet, le travail des actionnaires est conditionné par le contrôle d'un système de gouvernance qui oriente les décisions prises en adéquation avec l'intérêt de toutes les parties prenantes de l'entreprise. En raison de l'importance de cette nouvelle culture d'entreprise, plusieurs chercheurs des théories des firmes ont essayé de mettre en évidence la gouvernance au sein de l'entreprise. Nous enregistrons la définition proposée par Charreaux (1997): " Le système de gouvernement d'entreprise recouvre l'ensemble des mécanismes organisationnels qui ont pour effet de délimiter les pouvoirs et d'influencer les décisions des dirigeants, autrement dit, qui gouvernent leur conduite et définissent leur espace discrétionnaire ». La gouvernance au sein de l'entreprise est donc importante car elle contribue à l'amélioration de l'efficience et de la croissance économique. En 
effet, la gouvernance participe à encrer la notion de la confiance entre les différentes parties prenantes de l'entreprise, et également à la promotion de l'économie dans sa globalité. Outre les avantages micro et macro économiques obtenus suite à la mise en place d'un système de gouvernance efficace au sein de l'entreprise, il faut également mettre l'accent sur la nouvelle relation entre les propriétaires et les managers. II est tout à fait logique que la gouvernance d'entreprise aide à mieux schématiser cette relation. En effet, elle englobe, non seulement la relation managers-propriétaires, mais elle fait aussi référence aux différentes parties prenantes de l'entreprise. Pour mieux expliquer cette notion, on rappelle, plus exhaustivement, le rôle d'un système de gouvernance efficace, qui se résume autour des trois points suivants : la protection des droits des propriétaires, la transparence et la diffusion de l'information, la participation des propriétaires à la prise de décision.

- Protection des droits de propriétaires: la gouvernance de l'entreprise doit assurer le pilotage stratégique de l'entreprise et le contrôle permanent et effectif de la gestion par les managers, de telle manière à protéger les intérêts des propriétaires et à assurer la pérennité des entreprises. Elle doit reconnaitre les droits des différentes parties prenantes à la vie d'une société. Ainsi, la gouvernance d'entreprise contribue à la définition des objectifs stratégiques d'une entreprise, ainsi qu'à l'élaboration des moyens en vue des les atteindre, et en dernier lieu, elle opère un contrôle permanent sur les résultats obtenus.

- La transparence et la diffusion de l'information: Avec la mise en place d'un régime de gouvernement d'entreprise efficace, les actionnaires sont en droit d'avoir accès à l'information. En effet, ils doivent être informés, en temps opportun, de toutes les informations concernant l'entreprise, telles que la situation financière, et les résultats de l'entreprise. Ces informations doivent être exactes et ne doivent en aucun cas contenir des informations mensongères. D'autant plus, qu'ils doivent être informés des dates de réunions des assemblées générales, des décisions devant être prises lors de l'assemblée générale, du règlement de ces assemblées et des procédures de vote.

- Participation des actionnaires à la prise de décision : les actionnaires sont en droit de connaître les décisions prises par les managers (conseil d'administration), et éventuellement donner leurs avis sur ces orientations qui concernent la société. Ils ont ainsi le droit de participer aux assemblées générales et de voter sur le maintien d'une décision prise par le conseil d'administration ou le cas échéant l'abolir et voter une autre décision qui répond au mieux à leurs intérêts et qui participe efficacement à la croissance économique. Par conséquent, la gouvernance d'entreprise a pour rôle d'assurer un traitement équitable de tous les actionnaires (minoritaires comme majoritaires).

\subsection{La coopérative et les principes de coopération}

A partir des principes de la gouvernance d'entreprise que nous avons cités, ci-dessus, la gouvernance s'avère être une nouvelle forme de démocratie participative. Elle construit une culture de concertation, de coopération et de partenariat entre tous les agents économiques. De ce fait, elle permet à toutes les parties prenantes de l'entreprise, à prendre des décisions approuvées par la majorité et répondants à leurs intérêts communs. De plus, la gouvernance est une notion universelle, appliquée à toutes les organisations, quelles soient privées ou publiques. Cette application nous interpelle sur la pertinence du régime de gouvernance au sein des coopératives, plus précisément les coopératives du safran de Taliouine, objet de notre article et de notre seconde partie. Toutefois, en préliminaire à cette étude, nous mettons l'accent sur les coopératives au Maroc, sur les principes de coopération et sur le cadre législatif et juridique, à travers une lecture détaillée de la loi $n^{\circ}$ 112-12 qui est une refonte des dispositions de la loi $n^{\circ}$ 24-83 fixant le statut général des coopératives qui a été promulguée par le dahir $n^{\circ}$ 1-83-226 du 5 octobre 1984. Tout d'abord, il existe plusieurs définitions du mot coopérative, mais dans une vision de cadrer notre recherche sur les coopératives marocaines, nous avons choisie la définition proposée dans la loi $n^{\circ} 112-12$ à savoir : "La coopérative est un groupement de personnes physiques, qui conviennent de se réunir pour créer une entreprise chargée de fournir, pour leur satisfaction exclusive, le produit ou le service dont elles ont besoin et pour la faire fonctionner et la gérer en appliquant les principes fondamentaux définis à l'article 2 ci-après et en cherchant à atteindre les buts déterminés à l'article 3 de la présente loi. Des personnes morales remplissant les conditions prévues par la présente loi peuvent devenir membres d'une coopérative $»{ }^{1}$

A partir de cette définition, les personnes, devenus membre d'une coopérative, travaillent en équipe afin de réaliser un objectif commun à savoir l'amélioration de leurs situations socio-économiques, et le développement durable

\footnotetext{
${ }^{1}$ Extrait de la loi $n^{\circ} 112-12$
} 
de leur communauté. Cette situation implique un esprit coopératif entre les membres de la coopérative pour améliorer la qualité du produit fourni et maximiser la production. Toutefois, afin d'assurer la pérennité des coopératives, il existe des principes édictées par l'article 2 de la loi $n^{\circ}$ 112-12 qui sont en adéquation avec les sept principes internationaux de coopération qui ont été formulés par l'Alliance coopérative internationale. On rappelle, ci-après, les principales idées :

- Adhésion volontaire et ouverte à tous: la coopérative est ouverte à toute personne qui désire y adhérer à condition qu'elle remplisse les conditions de fond arrêtées par les constituants de cette dernière en raison de son activité. Par ailleurs, le retrait d'un des membres de la coopérative est possible à condition de ne pas porter préjudice à son fonctionnement par un retrait intempestif.

- Droit à la prise de décision : les membres de la coopérative sont tous égaux. Par conséquent, ils ont le droit de participer à l'établissement des politiques et à la prise de décisions

- Partage équitable des recettes: les excédents des recettes sont répartis de façon équitable entre les membres, au prorata de la participation de chacun d'entre eux. Par ailleurs, les excédents mis en réserve sont alloués au développement de la coopérative.

- Éducation et formation des membres: les membres de la coopérative (employés, dirigeants élus et gestionnaires) bénéficient de séances de formation afin qu'ils puissent contribuer efficacement au développement de leur coopérative.

- Coopération entre les coopératives: les coopératives ont la possibilité de collaborer avec d'autres coopératives œuvrant sur le plan national comme international.

D'un autre côté, il faut rappeler que la coopérative est une entité indépendante financièrement. Le statut juridique des coopératives tel qu'il a été énoncé au niveau de l'article 4 de la loi $n^{\circ} 112-12$ stipule que : « les coopératives sont des personnes morales jouissant de la pleine capacité juridique et de l'autonomie financière». A l'issue de cette étude de la loi $n^{\circ} 112-12$, nous concluons que la coopérative est une organisation autonome et démocratique basée sur l'esprit d'entraide, de coopération et de confiance.

\section{Résultats et discussions}

Dans cette partie, nous portons notre attention sur les coopératives du safran de Taliouine, afin de répondre à la question primordiale de cet article comment une bonne gouvernance des coopératives peut elle participer à la promotion de la commercialisation de leurs produits. Tout d'abord, nous étudions l'existence d'un système de gouvernance au sein de ces coopératives et ultérieurement l'efficacité de ce régime au regard des résultats obtenus en matière de commercialisation du produit du Safran. Nous rappelons que notre étude a été élaborée suite aux résultats recensés lors de notre enquête sur le terrain auprès des coopératives du Safran au niveau de Taliouine au Maroc, à travers des entretiens semi-directifs avec les membres et dirigeants de ces organisations solidaires.

Nous voulons, tout d'abord, partager un point très important qui nous a le premier marqué, lors de notre étude empirique. II s'agit de souligner que les responsables des coopératives sont conscients de l'importance de la contribution d'un régime de gouvernance efficace au sein de leurs coopératives. Ils comprennent mieux, à présent, l'impact du gouvernement d'entreprise sur leur productivité et leur compétitivité. Cette prise de conscience sur la nécessité de la mise en place d'une gouvernance efficace par les membres, est la résultante d'un effort considérable des dirigeants élus des coopératives à développer des nouvelles compétences et connaissances, à travers un système de formation continue fournie à l'ensemble des membres des coopératives.

La présentation de la sphère socio-économique de la région expliquera mieux la pertinence des décisions prises par les coopératives en matière de création, d'éducation et de formation. Les membres des coopératives du Safran de Taliouine, sont pour la majorité, des habitants des environs de cette ville du sud du Maroc. Ces habitants vivent principalement de la culture du safran. Une production qui est, en grande partie, vendue sur le marché informel, rendant par conséquent, ces habitants à la merci des intermédiaires du secteur. D'autant plus, la plupart de ces agriculteurs sont des personnes peu scolarisés. Par conséquent, ils sont plus susceptibles à être trompés par les revendeurs du secteur informel, au regard de la méconnaissance des agriculteurs de la valeur réelle du safran sur le marché national et mondial.

A partir de ce constat, les coopératives ont vu le jour pour garantir un développement durable à la région de Taliouine pour participer à l'amélioration du niveau de vie des agriculteurs. Ces coopératives ont réuni l'ensemble des agriculteurs de la région, leur offrant ainsi, un lieu où ils pourront vendre leur produit avec la garantie d'être payés équitablement. En effet, cette concentration de production permet aux coopératives de maîtriser la qualité du Safran à travers la mise en place de séances de formation allouées aux agriculteurs. Ces formations ont pour but d'assister les 
agriculteurs, tout au long du processus de production, afin de leur faire connaître les bonnes démarches à suivre en matière d'irrigation, de culture, de la récolte et du stockage du safran. Les nouvelles connaissances, ainsi acquises, permettent aux agriculteurs de produire un Safran de haute qualité, lui octroyant alors un avantage compétitif sur le marché local, national et international. D'autant plus que la coopérative apporte aux agriculteurs, une structure avec un conseil d'administration qui possède les compétences requises en matière de gestion de la coopérative, de conclusion de contrat avec les différents partenaires de la coopérative.

Nous nous focalisons, à présent, sur l'efficacité de la gouvernance au sein des coopératives du Safran de Taliouine. A travers notre étude empirique, nous avons étudié le respect des trois principes de la bonne gouvernance que nous avons expliqué lors de la première partie de ce texte.

\subsection{Protection des droits des membres :}

Les coopératives, de part leurs statuts et leurs objectifs, assurent la protection des intérêts des membres. Cet acquis est mieux expliqué par l'esprit d'entraide et de coopération entre les membres, déclencheur primordial de la création de la coopérative du Safran. Les membres, à savoir les agriculteurs, ont tous intérêt à maintenir la croissance de leur organisation, qui est leur source primordiale de revenus. D'autant plus que le cadre législatif qui coiffe les coopératives au Maroc a prononcé plusieurs organes administratifs, qui ont pour mission de garantir les intérêts des coopérateurs. La coopérative, selon sa taille et son chiffre d'affaire, est gérée soit par un conseil d'administration, un directeur ou un gérant de la coopérative. Ces derniers assument explicitement la responsabilité de l'administration saine et prudente de la coopérative. Ils revoient périodiquement son fonctionnement dans une perspective d'amélioration continue et veille à la protection du patrimoine du mouvement, de ses membres et de ses clients. En parallèle aux gestionnaires de la coopérative, un comité de surveillance existe au sein de chaque entité. Ce dernier exerce le contrôle permanent sur la gestion du conseil d'administration ou des gérants. Par la suite, il élabore un compte rendu contenant ses observations sur la gestion de la coopérative.

Afin de garantir au mieux les intérêts des coopérateurs, le législateur marocain a instauré l'office du développement de la coopération, qui a pour mission de s'assurer que les coopératives sont gérées conformément aux textes réglementaires en vigueur. A cet effet, l'office du développement de la coopération a l'aptitude de provoquer une assemblée générale extraordinaire pour prendre les mesures nécessaires afin de remédier à toute anomalie constatée lors de ses enquêtes. Toutefois, nous avons relevé quelques pratiques qui nuisent au maintien des intérêts des agriculteurs. Le premier inconvénient réside dans le système de commercialisation adoptée par les coopératives. Ce système repose sur le paiement tardif des producteurs. En effet, le paiement du producteur est conditionné par la vente effective du produit, entraînant ainsi un retard de paiement pour ce dernier. Cette situation est en contradiction totale avec l'objet même de la coopérative qui est d'aider l'agriculteur à améliorer sa situation socio-économique. Par conséquent, le maintien de cette pratique nuit considérablement à la relation producteur-coopérative, à travers le manque de confiance entre l'agriculteur et la coopérative. Cette situation peut ainsi freiner l'adhésion de nouveaux membres qui opteront de vendre leur production au niveau du secteur informel.

Ce constat suscite des interrogations sur l'option de cette politique de commercialisation. Une question se pose : pourquoi les dirigeants des coopératives ne changent-ils pas de politique. L'explication fournie par les coopératives est le défaut d'un fond de roulement important qui permet de payer les agriculteurs immédiatement. Toutefois, cette argumentation n'est pas tolérée actuellement, surtout dans un environnement caractérisé par les nouvelles technologies et l'ouverture des marchés. A cet effet, nous nous sommes focalisés sur les méthodes utilisées par les coopératives pour assurer la vente de leurs produits. Les coopératives comptent pour la plupart sur la vente directe de leurs produits. Toutefois, comme Taliouine est une région montagneuse, cette situation rend ainsi l'accès difficile aux coopératives des villages. D'autant plus que les coopératives sont méconnues du grand public en raison du manque de communication sur ces entités et sur leurs produits. D'autres coopératives plus au moins structurées, font de la prospection de la clientèle à travers la participation aux salons et aux foires. Ce sont des occasions qui leur permettent de vendre leurs produits sur place et de gagner de nouveaux clients (surtout dans les manifestations internationales telles que le SIAM2). D'autres coopératives sont plus au moins connus sur le net.

A l'issue de ce constat, il paraît intéressant de réfléchir à des solutions permettant de garantir les intérêts des agriculteurs et la croissance de la coopérative. Les coopératives, dans le cadre de coopération avec d'autres coopératives moins cloisonnées, installées au niveau national comme international, peuvent conclure des accords de

2 Salon International de l'agriculture du Maroc 
partenariat, visant à mieux communiquer leurs produits dans ses régions. II s'agit de création de bureaux de représentation, qui seront le lien entre ces coopératives cloisonnés et la clientèle éventuelle. Les coopératives peuvent également concentrer leurs efforts à nouer contact avec des sociétés de manufacturation utilisant le safran comme matière première dans leurs productions. Des accords avec des agences de voyages nationales et internationales opérant dans le tourisme équitable est une autre alternative. Ces initiatives auront pour objet de créer un porto folio de clients susceptibles d'acheter le produit dans l'immédiat.

\subsection{Transparence et diffusion de l'information :}

L'examen de ce point au niveau des coopératives safranières de Taliouine nous a permis de constater le respect de ce principe. En effet, des réunions périodiques sont organisées au sein des coopératives. Par exemple, la coopérative invite tous ses membres pour fixer les modalités de commercialisation du safran, à travers les deux éléments suivants : la fixation de la quantité mise en vente sur le marché et la détermination du prix d'achat. A la fin du mois de décembre de chaque année, une réunion est organisée entre les membres de la coopérative afin de décider de la quantité du safran apportée par chaque membre à la coopérative. Cette décision est importante car il est difficile de d'acheter toute la quantité produite par les agriculteurs, et ce à défaut du fond de roulement et de clientèle immédiate pour acheter le safran.

Ainsi, lors de cette réunion, un consensus est établi sur la quantité du premier lot de safran qu'apportera chaque producteur. Cette quantité dépend de la capacité de production de l'agriculteur. Pour le petit producteur, la quantité du lot varie de 250 à 500grammes. Pour les grands producteurs, le lot peut atteindre 1 à $2.5 \mathrm{~kg}$. Ensuite, une deuxième réunion est effectuée pour la détermination du prix d'achat du safran. Afin de déterminer le prix d'achat, la coopérative effectue une comparaison avec le prix d'achat dans le marché traditionnel (le souk). Si par exemple, le safran est vendu à 15 dirhams le gramme au marché, la coopérative propose 4 à 5 dirhams de différence en plus. Le consensus sur le prix d'achat nécessite du temps car les producteurs recherchent à gagner le plus possible.

Après avoir décidé de la quantité et du prix d'achat du safran, la coopérative donne aux producteurs un délai de 8 à 15 jours maximum pour apporter le premier lot de safran séché. La présence du conseil à ce moment est obligatoire. La coopérative conditionne le safran dans des boites qui portent le code du producteur, son nom, le lot et l'année de production pour assurer la traçabilité. Ces boites sont conservées dans des coffres forts. La gestion de stock utilisée est la méthode FIFO. Des échantillons sont gardés par la coopérative pour justifier que le produit de la coopérative est pur et sans défaut en cas d'éventuelles réclamations.

Un bon de réception est rempli pour chaque producteur contenant le nom, le code, la date où il a apporté le safran, la quantité, le prix unitaire et le prix total. La coopérative garde ce reçu jusqu'au paiement du producteur (avec le numéro de chèque et la copie du chèque) avec signature de l'agriculteur. Par conséquent, cette démarche a pour but de garantir la transparence des transactions au sein de la coopérative.

En réponse au respect du droit d'accès à l'information, la loi n ${ }^{\circ} 112-12$ dans son article 25, a octroyé pleinement ce pouvoir aux adhérents de la coopérative. En effet, le membre coopérateur peut à tout moment, consulter tous les documents administratifs, comptables et financiers de la coopérative. On cite par exemple, les états de synthèse, le rapport du conseil d'administration, le rapport du ou des commissaires aux comptes et les procès verbaux des assemblées générales. Le cadre législatif a également assuré ce droit même aux membres non scolarisés. En effet, ces derniers peuvent recourir à l'aide d'un conseiller lors de la consultation de ces documents.

\subsection{Participation à la prise de décision :}

Les coopératives tiennent annuellement, des assemblées générales ordinaires, auxquelles sont invités tous les membres de la coopérative. Lors de ces assemblées, il est question de prendre des décisions sur plusieurs sujets intéressants la coopérative. En effet, la loi a explicitement détaillée les principaux sujets dont se prononcent les assemblées générales. Elle est l'occasion de remettre en cause le travail du conseil d'administration. A cet effet, les coopérateurs ont le droit de vote sur toute décision intéressant la coopérative, qui va de la remise en cause des comptes annuels à la désignation des membres du comité de surveillance, des commissaires aux comptes et du conseil d'administration. Toutefois, un élément empêche le respect de ce principe au sein des coopératives safranières de Taliouine, à savoir l'analphabétisme des agriculteurs. Cet inconvénient induit que les membres n'ont pas de connaissance en matière de gestion et de prise de décision. Par conséquent, les seules décisions prises par les coopérateurs sont celles de l'approbation d'adhésion de nouveaux membres, la répartition des bénéfices aux membres selon la quantité apportée. Les membres ont des parts 
dans la coopérative mais l'excédent est distribué selon la quantité apporté et le travail effectué au sein de la coopérative. Les membres sont appelés un par un pour leur donner leur part du bénéfice. Ces bénéfices peuvent être réutilisés après un accord de la part de tous les membres (un vote) pour permettre à la coopérative de financer ses activités.

Certes, le comité de surveillance des coopératives minimisent le risque de mauvaise gestion. En effet, les dirigeants des coopératives sont surveillés de près par l'office de développement de la coopération, qui rend compte régulièrement de l'exercice de leurs tâches. En parallèle, il existe que des sanctions pénales ont été édictées par la loi en cas d'observation de toute anomalie de gestion ou de tout écart de bonne conduite par les dirigeants des coopératives, nuisant ainsi aux intérêts des coopérateurs. Toutefois, la coopérative doit plus investir dans l'éducation des coopérateurs sur leurs droits coopératifs. Des séances de formation sont organisées en concertation avec l'Office de développement des coopérations, afin de mettre à niveau les membres des coopératives. En effet, les coopérants doivent être des agents qui participent efficacement à la prise des décisions stratégiques des coopératives. Comment peut-on développer la rentabilité des coopératives, si les principaux membres ne sont pas conscients de leurs droits et de leurs devoirs. Le coopérant n'est pas seulement un fournisseur, il représente également un capital pour la coopérative dans le sens où il est membre actif et soucieux de remettre en cause continuellement les résultats obtenus, et de participer à l'élaboration des orientations stratégiques. Par conséquent, il faut mettre l'accent sur la mise à niveau des membres et des dirigeants des coopératives afin d'atteindre les objectifs escomptés. II faut penser à intégrer au sein de ces entités de jeunes compétences de la région de Taliouine, qui ont acquis de par leurs cursus universitaires, des connaissances en matière de techniques de gestion et de commercialisation des produits de terroir. Ils seront les formateurs relais auprès des membres des coopératives.

\section{Conclusion}

En conclusion, l'étude de l'efficacité du système de gouvernance au sein des coopératives safranières de Taliouine nous a permis de recenser des points forts et aussi des points d'amélioration. Nous résumons les points forts du système à travers les points suivants :

- Un cadre législatif répondant aux standards nationaux en matière de coopération, qui protège les intérêts des coopérants à travers la multiplicité des organes de contrôle édictées par la loi ;

- Confiance entre les coopérants et ancrage de l'esprit coopératif ;

- Les coopérateurs ont droit à toute information relative à leur organisation. Un droit qui est préservé même en cas d'analphabétisme du coopérant ;

- La transparence et traçabilité constatée dans les processus d'achat du safran aux agriculteurs (prix et quantité) ;

- La commercialisation du safran avec un prix mieux valorisé que sur le marché traditionnel ou informel ;

- La protection des intérêts des coopérants.

En ce qui concerne les axes nécessitant des améliorations afin de garantir l'efficacité du régime de gouvernance au sein des coopératives du safran de Taliouine, nous les résumons à travers les points ci-après :

- Refonte de la stratégie de commercialisation du produit safran par les coopératives à travers la mise en place d'une structure de prospection de la clientèle et de communication autour de la coopérative et de son produit ;

- Favoriser la coopération entre les coopératives safranières, à travers un regroupement qui leur offrira plus d'opportunités de croissance ;

- Prodiguer des formations aux agriculteurs en matière de gestion des coopératives, afin qu'ils participent efficacement à la prise de décision ;

- Assister les dirigeants des coopératives analphabètes et qui n'ont aucune compétence en matière de gestion ou d'administration d'une organisation telle que la coopérative, à développer les connaissances requises pour l'exécution de leurs fonctions.

- Développer davantage l'esprit coopératif au sein des adhérents et des dirigeants.

\section{References}

Alchian, A., \& Demsetz, H., (1972), «Production, Information Costs, and Economic Organization», American Economic Review, 62,777795.

Charreaux, G., (1991) «Structure de propriété, relation d'agence et performance financière», Revue Economique, 3(42), $521-552$. 
Charreaux, G., (1992). « Mode de contrôle des dirigeants et performance des firmes », Revue d'Économie Industrielle, Hors-Série, $n^{\circ}$ exceptionnel, 135-172.

Charreaux, G., (1993). « Conseil d'administration et pouvoirs dans l'entreprise », Revue d'Économie Financière, 31(31), 49-79.

Charreaux, G., (1998). « Le rôle de la confiance dans le système de gouvernance des entreprises », Économies et Sociétés, Sciences de Gestion, (8-9), p.47-65.

Clarke, E. (1971). «Multipart Pricing of Public Goods », Public Choice, 11, 17-33.

Desbrières, P. (1997). « Le point sur le gouvernement des entreprises », Revue Banque \& Marchés, juillet-août 1997, (29), 28-34.

Groves, Th. (1973). "Incentives in Teams", Econometrica, 41, 617-631.

Jensen M.C., \& Meckling, W.H. (1976). " Theory of firm, managerial behavior agency costs and ownership structure », Journal of Financial Economics, 3(4), 305-360.

Laffont J.J., \& Martimort D., (2001). The Theory of Incentives: the Principal-Agent Model. Princeton University Press, Oxford.

Laffont, J.J. (2000). «À propos de l'émergence de la théorie des incitations ». la Revue française de gestion 32(160), 177-190.

Ministère Délégué Auprès du Chef du gouvernement Chargé des Affaires Générales et de la Gouvernance, Royaume du Maroc. (2012). Note de présentation du projet de loi $\mathrm{n}^{\circ} 112$-12 relative aux coopératives au Maroc. Récupéré de http://www.sgg.gov.ma/Projet_loi_112-12_Fr.pdf

Rebérioux, A., (2003). «Gouvernance d'entreprise et théorie de la firme : Quelle(s) alternative(s) à la valeur actionnariale », Revue d'économie industrielle, 104(104), 85-110. 